\title{
Combining stigmergic and flocking behaviors to coordinate swarms of drones performing target search
}

\author{
Mario G. C. A. Cimino, Alessandro Lazzeri, Gigliola Vaglini \\ Department of Information Engineering \\ University of Pisa \\ Pisa, Italy \\ mario.cimino@unipi.it, alessandro.lazzeri@for.unipi.it, gigliola.vaglini@unipi.it
}

\begin{abstract}
Due to growing endurance, safety and noninvasivity, small drones can be increasingly experimented in unstructured environments. Their moderate computing power can be assimilated into swarm coordination algorithms, performing tasks in a scalable manner. For this purpose, it is challenging to investigate the use of biologically-inspired mechanisms. In this paper the focus is on the coordination aspects between small drones required to perform target search. We show how this objective can be better achieved by combining stigmergic and flocking behaviors. Stigmergy occurs when a drone senses a potential target, by releasing digital pheromone on its location. Multiple pheromone deposits are aggregated, increasing in intensity, but also diffused, to be propagated to neighborhood, and lastly evaporated, decreasing intensity in time. As a consequence, pheromone intensity creates a spatiotemporal attractive potential field coordinating a swarm of drones to visit a potential target. Flocking occurs when drones are spatially organized into groups, whose members have approximately the same heading, and attempt to remain in range between them, for each group. It is an emergent effect of individual rules based on alignment, separation and cohesion. In this paper, we present a novel and fully decentralized model for target search, and experiment it empirically using a multi-agent simulation platform. The different combination strategies are reviewed, describing their performance on a number of synthetic and real-world scenarios.
\end{abstract}

Keywords-swarm intelligence; small drone; stigmergy; flocking; target search

\section{INTRODUCTION AND MOTIVATION}

Small drones are unmanned aerial vehicles small enough to be man-portable. Recent advances in sensing technology encourage the use of small drones for military intelligence, reconnaissance, surveillance, traffic monitoring, forest fire localization, scientific surveys in dangerous conditions, border and harbor patrol, search and rescue, wildlife tracking, and so on [1]. Indeed, small drones can be equipped with selflocalization and sensing capabilities, to be used for information gathering tasks, such as target search or environmental sensing. More specifically, in target search small drones offer more potential, because they can perform tasks in highly inhospitable environments, where access to humans and large drones is limited or impossible, or in environments where medium and large drones can cause significantly more damage. Moreover, in tasks such as search and rescue, to accurately scan every available location on the area is also an inappropriate strategy. A more effective approach is to achieve a quick "survey" of the area, identifying key locations as quick as possible, and to better investigate only key locations that provided some circumstantial evidence. To complete this task with a single drone, both structure and control logic should be highly costly in terms of design, construction and maintenance. Moreover, a unique drone is vulnerable, because a single hardware or software fault may affect the whole system, and it is difficult to predict. Hence, a number of considerations support the use of small drones.

To take advantage of the above strategy, an important requirement is to avoid centralized control approaches, which frequently lead to exponential increases in communication bandwidth requirements and software complexity [2]. To solve problems cooperatively while maintaining scalability, application designers are investigating swarm intelligent methodologies. The main inspiration for swarm drones comes from the observation of social animals, such as ants, bees, birds, and fishes, exhibiting a sort of collective intelligence which appears to achieve complex goal through simple rules and local interactions [3]. The main benefits of swarm drones are: (i) robustness, for the ability to cope the loss of individuals; (ii) scalability, due to the ability to perform well with different group size; (iii) flexibility, thanks to the ability to cope with a broad spectrum of different environments and tasks. For this purpose, each individual of the swarm: (i) acts with a certain level of autonomy (ii) performs only local sensing and communication; (iii) operates without centralized control or global knowledge, and (iv) cooperates to achieve a global task.

From a structural standpoint, we assume that each small drones is provided with the following equipment: (a) wireless communication capability for sending and receiving information from the ground station; (b) self-location capability, e.g. based on global position system (GPS) and inertial technology, returning the coordinates of its current location; (c) one or more target sensing technology, capable of capturing samples in the area over which it flies; (d) processor with limited computing capability; (e) obstacle avoidance capability, i.e., locally managed detection and steering to avoid flying towards surrounding barriers and drones.

A fundamental swarm coordination mechanism is markerbased stigmergy [4]. With stigmergy, individuals leave 
information in the environment in the form of pheromones, volatile substances diffused locally and staying temporarily for other individuals that properly react and modify their behavior [5]. Simulated pheromones can be used to coordinate groups of drones for various tasks. We assume that the pheromone map is built and maintained on each region of the search space, and made available at the ground station for drones as a "remote brain" capability [6].

When the sensing system of a drone determines a potential target, it has to enable the cooperation of its swarm. Indeed, in case of a complex target, there are likely to be multiple pieces of target within the surrounding region. In case of a simple target, it is supposed that their distribution is not uniform, and then, again, there are likely to be other simple targets within the surrounding region. For this purpose, the drone releases a particular amount of pheromone with the location of the sensed target, whose diffusion acts as an attractive potential on neighboring drones. As an effect, other drones helps in sensing surrounding targets or piece of them, thus enabling an emerging behavior of the swarm that aggregate a considerable amount of pheromone for each new sensed piece of target. Since pheromone evaporate over time and already sensed targets do not activate additional pheromone, after a certain time the pheromone intensity cannot be reinforced in an explored region, and in practice disappears.

To be attracted by pheromone trails, the available drones should be spatially organized into a number of swarms. Each member of a swarm has approximately the same heading of the other members, and attempts to remain in range between them. For this purpose, the structural dimensions of the pheromone should take into account the average size of a swarm (or vice versa). Otherwise, a highly diffused or poorly evaporated pheromone could attract disproportionate resources on a single target, thus interfering with the progressive development of the emergent behavior. In contrast, a poorly diffused or highly evaporated pheromone could not be sensed at all. Flocking is a strategy to allow the self-organization of drones into a number of swarms. Flocking behavior is an emergent effect of individual rules based on alignment, separation and cohesion [7]. Indeed, with alignment rules the drones tends to move in the same direction that nearby drones. With separation rules, the drone keeps a minimum distance able to provide the drone with flexibility when moving in the swarm, and for a better exploration. Finally, with cohesion rules the drone tends to move towards the swarm.

In our approach, stigmergy and flocking are two emergent behavioral patterns which should work in conjunction with other basic behavioral patterns of the drone, such as obstacle and boundary avoidance. The process of designing a combination strategy is bottom-up and consists in finding the right setting at the micro-level (agent-level) in order to obtain a coherent emergent behavior at macro-level (swarm-level or even global-system level) [8]. In this paper, the different combination strategies are reviewed and tested empirically with both synthetic and real-world scenarios, with obstacles having irregular complex shapes. For this purpose, we adopted a multi-agent simulation platform with the possibility of importing environments with obstacles and targets sampled from real landscapes.
The paper is structured as follows. Section II briefly characterizes related work. In Section III, the design of the proposed model is covered. Experimental studies are detailed in Section IV. Section V draws conclusions and future works.

\section{RELATED WORK}

The goal of this section is to briefly characterize the main approaches and results in the literature on stigmergic mechanisms coordinating swarms of small robots to perform target search or similar tasks. The published works in the field can be distinguished into three categories: (a) using a physical substance as a pheromone, which is necessarily transmitted in an indirect way between robots, by means of the physical environment; (b) using a digital pheromone, transmitted via direct communication between robots; (c) using a digital pheromone, transmitted via an indirect communication between robots. The latter is the category of our approach.

In [9] the authors use a swarm of robots releasing physical substance as a repulsive pheromone, for environment exploration. In particular, robots act combining three basic behaviors, with decreasing priority: wall avoiding, pheromone coordination, and random walk. Actually there are various approaches in the literature using physical pheromones, because they do not require a computational structure. Although real pheromones are not usable with aerial vehicle, they can be simulated. Thus, this type of research can be interesting to digitally model new types of stigmergy.

An example of stigmergic coordination between drones using direct communication is presented in [10], where the author focuses on automatic target recognition. Potential target are marked by drones, which also communicate the gossiped pheromone to nearby drones, with probability inversely proportional to the distance from the source. The proposed stigmergic schema employs also repulsive pheromone, as a negative feedback, when a predefined number of drones identify the same target. A disadvantage of such scheme is that the bandwidth required goes into an exponential explosion as the population grows. Moreover, to avoid redundancy in target evaluation each UAV has to maintain in memory the state of each potential and confirmed target. In this way, the direct communication in the swarm should be strongly limited [11].

A swarm coordination schema with indirect coordination is proposed in [4]. Here the coordination of a swarm of vehicles is based on digital pheromones maintained in an artificial space called pheromone map and composed by an arbitrary graph of place agents, i.e., intermediate control nodes. There are two classes of agents which deposit, withdraw, and read pheromones, i.e., walkers and avatars. A walker agent aims to make movements and action decisions, whereas avatars collect location information to make estimates when sensor information is not available. The schema has been applied to a range of scenarios, among which target acquisition. An important problem of this approach is that the exploration depends on the initial state of deploy of the swarm. Moreover, this model does not consider complex targets but only simple targets with no reciprocal relationships. 


\section{DESIGN OF ENVIRONMENT DYNAMICS AND DRONE BEHAVIOR}

This section explains the logic structure of both the environment and the drones, together with their parametric setting.

\section{A. The environment structure and the pheromone dynamics}

Without loss of generality, we assume that the environment is constrained to a specific area. We superimpose to this area a grid consisting of $C^{2}$ cells, each identified by a pair $(x, y)$ of coordinates, with $x, y \in[1, \ldots, C]$. The actual size of the area and the number of squares depend on the specific application domain. Fig. 1 shows some basic scenario of the marking process. The levels of pheromone intensity are represented by different grey gradations: the darker the gradation is, the higher the intensity.

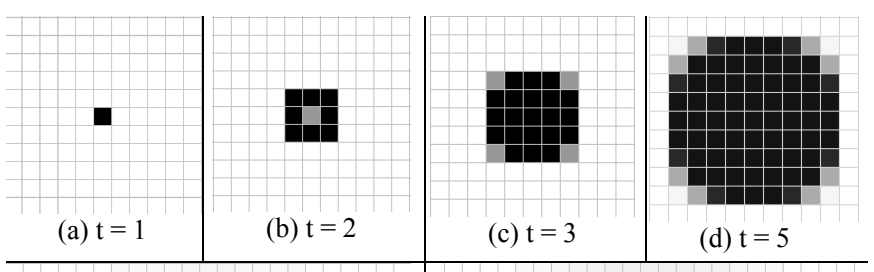

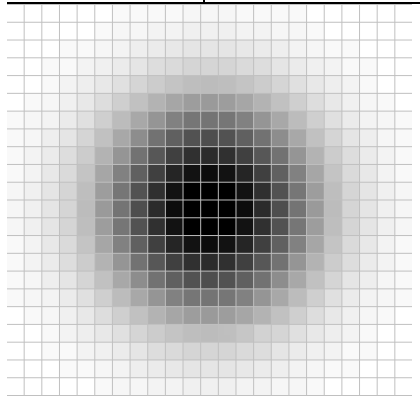

(e) $\mathrm{t}=20$

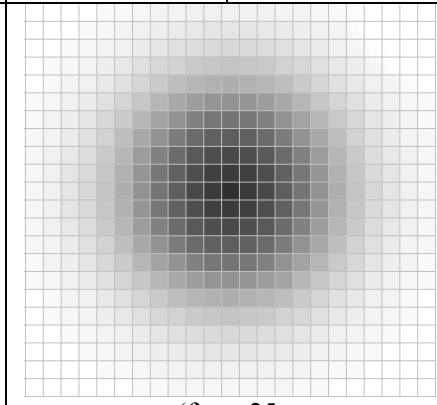

(f) $t=25$
Fig. 1. Basic scenario of pheromone dynamics: (a) releasing; (b) mainly diffusing; (c-d) diffusing and evaporating; (e-f) mainly evaporating.

More specifically, see Fig. 1: (a) a single pheromone intensity $I$ is released; (b) at the first step, the pheromone is mainly diffusing (moving) to the nearby cells, with a constant diffusion rate $\delta \in[0,1]$; (c-d) the pheromone is diffusing and evaporating; by evaporating, pheromone decreases its intensity over time; it is ruled by the constant rate $\varepsilon \in[0,1]$; (e-f) the pheromone is mainly evaporating. More formally, the pheromone intensity $p$ released at the instant $t$ on the cell $(x, y)$ is then characterized by the following dynamics:

$$
p_{x, y}(t)=\varepsilon \cdot\left[(1-\delta) \cdot p_{x, y}(t-1)+\Delta p_{x, y}(t-1, t)+d_{x, y}(t-1, t)\right]
$$

where $(1-\delta) \cdot p_{x, y}(t-1)$ represents the amount remaining after diffusion to nearby cells, $\Delta p_{x, y}(t-1, t)$ the additional deposits made within the interval $(t-1, t]$, and $d_{x, y}(t-1, t)$ the input pheromone diffused from all the nearby cells. The latter can be formally calculated as:

$$
d_{x, y}(t-1, t)=\frac{\delta}{8} \sum_{\substack{i=-1 \\(i, j) \neq(0,0)}}^{1} p_{x+i, y+j}(t-1)
$$

since each of the 8 neighbor cells propagates the portion $\delta$ of its pheromone to the cell $(x, y)$ at each update cycle. The total amount in (1) is also multiplied by $\varepsilon$ to take into account evaporation.

\section{B. The drone behavior}

Basically, at each update cycle (hereafter called tick), the drone behavior is structured into a three-layer logic, where each layer implements one basic behavior: random fly (exploration) is the behavior corresponding to the lowest priority layer, while the pheromone-based coordination (exploitation) is the middle layer, and the objects/boundary avoiding and the pheromone releasing behaviors are realized by the highest priority architectural level.

Fig. 2 shows an overall representation of the drone behavior, using a UML activity diagram. Here, a roundedcorner box represents an activity (a procedure), a rectangular box represents an input/output data object (a parameter), solid and dashed arrows represent control and data flows, respectively. The diagram is also structured into three horizontal lanes, representing physical or logical components.

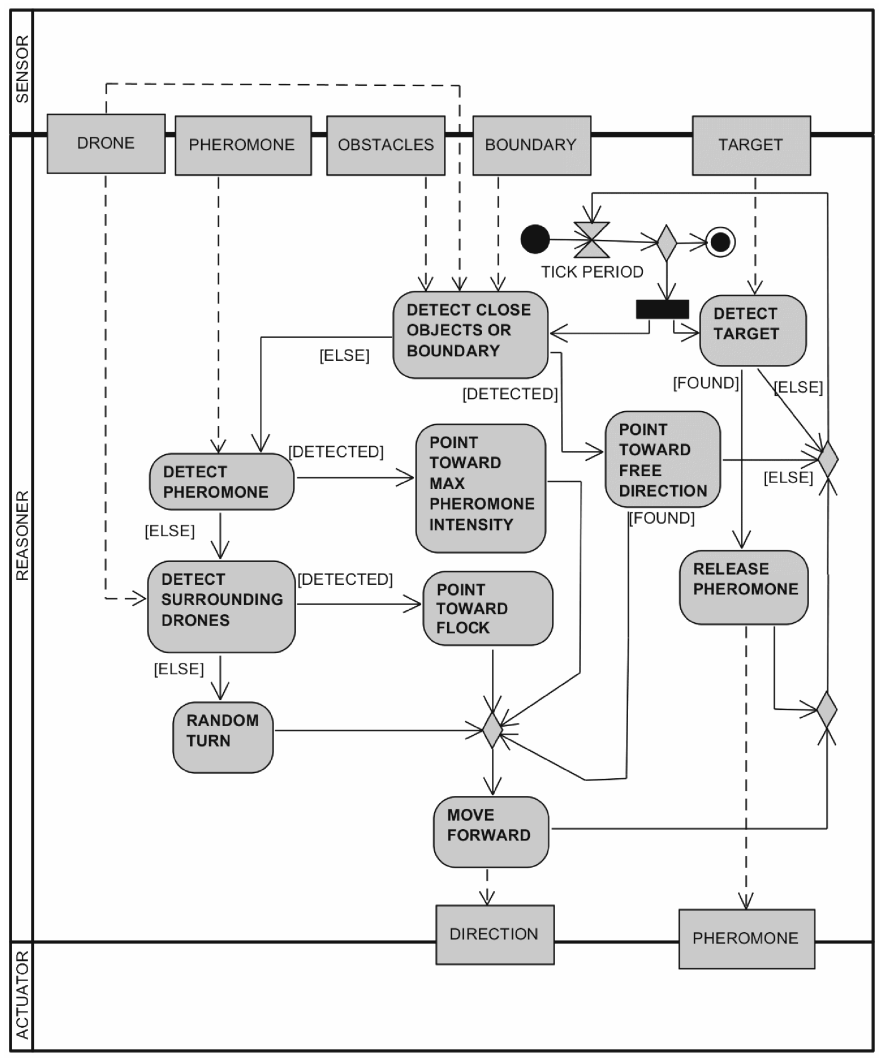

Fig. 2. Overall representation of the drone behavior.

More precisely, every tick period the drone performs in parallel: (a) the target detection, in which case it releases pheromone; (b) the objects and boundary detection. If a close object (i.e., obstacle or drone) is detected, the drone points toward a free direction. If a free direction is found, it moves forward. Otherwise, if there are no close objects detected, the drones try to sense pheromone and, if detected, points toward 
the maximum intensity of it (the drone is able to detect pheromone at the pheromone sensing distance $\pi$ ). Differently, if pheromone is not detected, the drone tries to detect surrounding drones in order to point toward the flock. Finally, if there are no surrounding drones, it performs a random turn and move forward.

In the flocking behavior, the drone takes into account only drones within a flock visibility radius $(\rho)$. Fig. 3 shows the main flocking parameters. More specifically, Fig. 3a represents the separation behavior: drones close to others have to separate for better exploration; thus, if a drone senses another drone closer than the flock mobility distance $(\mu)$, it turns by an angle $\sigma$ (flock separation angle).

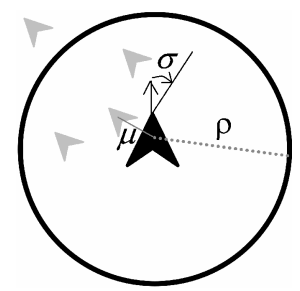

(a) Separation

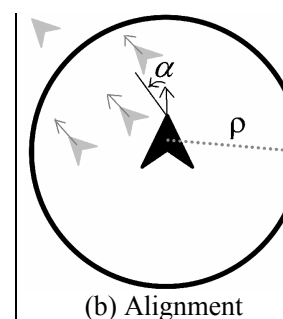

(b) Alignment

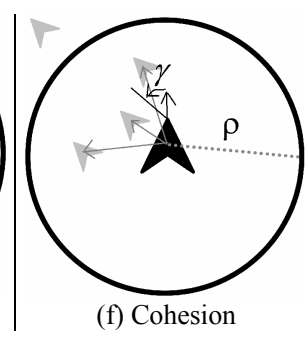

(f) Cohesion
Fig. 3. Flock visibility radius and other parameters in flocking behavior.

Fig. 3b shows the alignment behavior: the drone calculates the average direction of the drones in the flock visibility radius and turns by an angle $\alpha$ (flock alignment distance) to conform its direction to the flock direction. Fig. 3c illustrates the cohesion behavior: isolated drones do not sense digital pheromone, and this prevent cooperation; thus, drones tend to stay in proximity; for this reason, the drone calculates the barycenter of the drones in the flock visibility radius and turns by an angle $\gamma$ (flock cohesion angle) towards the barycenter.

By means of a reactive behavior, a drone is able to sense and avoid close obstacles, drones, and boundary (objects) at the distance $o$ (object sensing distance) on its trajectory. Here the drone horizontal speed $(v)$ has been modeled, when it moves forward. If an object is in its collision trajectory, the drone finds a free trajectory or stops moving. It randomly chooses to turn left or right to avoid the obstacle. Then, it turns one degree by one and checks the new trajectory. If the drone finds a free trajectory, before turning by 180 degrees it moves, otherwise it stops for one tick. It is worth noting that a drone flies at 1 meter height from the ground, and then we consider buildings and trees as obstacles to be modeled.

Actually, the boundaries of the total navigation area can be calculated by using the drone position and comparing it to the perimeter. This can be done by the ground station, which simulates a "wall" so as to avoid losing the drone. Finally, when the drone is in the basic behavior (random fly), it randomly turns by an angle smaller than $\theta$ (maximum rand-fly turn angle).

For the reader's convenience, Table I summarizes the main structural and behavioral parameters of the model, with their range and their value set.
TABLE I. STRUCTURAL AND BEHAVIORAL PARAMETERS

\begin{tabular}{|c|c|c|c|c|}
\hline Parameter & Description (unit measure) & Section & Range & Set $v$. \\
\hline$v$ & Drone horizontal speed $(\mathrm{m} / \mathrm{s})$ & III.B & $(0,15)$ & 1 \\
\hline$\theta$ & Drone max rand-fly turn angle $\left({ }^{\circ}\right)$ & III.B & $(0,180)$ & 90 \\
\hline$o$ & Drone object sensing distance $(\mathrm{m})$ & III.B & $(0,5)$ & 5 \\
\hline$\rho$ & Flock visibility radius (m) & III.B & {$[0,50]$} & 10 \\
\hline$\mu$ & Flock mobility distance (m) & III.B & {$[0,5]$} & 3 \\
\hline$\sigma$ & Flock separation angle $\left(^{\circ}\right)$ & III.B & $(0,180)$ & 20 \\
\hline$\alpha$ & Flock alignment angle $\left({ }^{\circ}\right)$ & III.B & $(0,180)$ & 8 \\
\hline$\gamma$ & Flock cohesion angle $\left(^{\circ}\right)$ & III.B & $(0,180)$ & 5 \\
\hline$I$ & Pheromone release intensity & III.A & $(0, \infty)$ & $50 \mathrm{~K}$ \\
\hline$\delta$ & Pheromone diffusion rate $(\%)$ & III.A & {$[0,1]$} & 0.90 \\
\hline$\varepsilon$ & Pheromone evaporation rate $(\%)$ & III.A & {$[0,1]$} & 0.95 \\
\hline$\pi$ & Pheromone sensing distance $(\mathrm{m})$ & III.B & $(0, \infty)$ & 1 \\
\hline
\end{tabular}

\section{EXPERIMENTAL STUDIES}

The proposed model has been implemented on $\operatorname{Net} \operatorname{Logo}^{1}$, a leading simulation platform for swarm intelligence. To assess the effectiveness of the proposed approach, we have evaluated the performance of the model on the three basic behaviors: random fly ("R"), stigmergic behavior ("S"), stigmergic and flocking behavior ("S+F"). For each experiment, 10 trials have been carried out, by using as a performance indicator the time needed to detect the $95 \%$ of targets in the scenario. We tested the model on 5 different scenarios. We also determined that the resulting performance indicator samples are well-modeled by a normal distribution, using a graphical normality test. Hence, we calculated the $95 \%$ confidence intervals. Table II summarizes the characteristics and the results for each scenario, in the form "mean \pm confidence interval".

TABLE II. NUMERICAL RESULTS

\begin{tabular}{|c|c|c|c|c|c|}
\hline Scenario & $\begin{array}{c}N^{\circ} \text { of targets } / \\
\text { clusters }\end{array}$ & $\begin{array}{c}\text { Type } / n^{\circ} \text { of } \\
\text { obstacles }\end{array}$ & Drones & \multicolumn{2}{|c|}{$\begin{array}{c}\text { Completion time } \\
\text { (ticks) }\end{array}$} \\
\hline \multirow{3}{*}{ Field } & \multirow{3}{*}{$50 / 5$} & \multirow{3}{*}{$\begin{array}{l}\text { Trees: } 0 \\
\text { Build: } 0\end{array}$} & \multirow{3}{*}{80} & $\mathrm{R}$ & $1756 \pm 178$ \\
\hline & & & & $\mathrm{S}$ & $802 \pm 160$ \\
\hline & & & & $\mathrm{S}+\mathrm{F}$ & $689 \pm 142$ \\
\hline \multirow{3}{*}{ Forest } & \multirow{3}{*}{$20 / 1$} & \multirow{3}{*}{$\begin{array}{l}\text { Trees: } 400 \\
\text { Building: } 0\end{array}$} & \multirow{3}{*}{80} & $\mathrm{R}$ & $2378 \pm 512$ \\
\hline & & & & $\mathrm{S}$ & $744 \pm 194$ \\
\hline & & & & $\mathrm{S}+\mathrm{F}$ & $677 \pm 68$ \\
\hline \multirow{3}{*}{ Urban } & \multirow{3}{*}{$110 / 2$} & \multirow{3}{*}{$\begin{array}{l}\text { Tree: } 0 \\
\text { Building: } 7\end{array}$} & \multirow{3}{*}{40} & $\mathrm{R}$ & $1448 \pm 106$ \\
\hline & & & & $\mathrm{S}$ & $861 \pm 148$ \\
\hline & & & & $\mathrm{S}+\mathrm{F}$ & $800 \pm 73$ \\
\hline \multirow{3}{*}{$\begin{array}{l}\text { Rural } \\
\text { Mines }\end{array}$} & \multirow{3}{*}{$28 / 28$} & \multirow{3}{*}{$\begin{array}{l}\text { Tree: } 281 \\
\text { Building: } 3\end{array}$} & \multirow{3}{*}{200} & $\mathrm{R}$ & $724 \pm 83$ \\
\hline & & & & $\mathrm{S}$ & $694 \pm 88$ \\
\hline & & & & $\mathrm{S}+\mathrm{F}$ & $666 \pm 68$ \\
\hline \multirow{3}{*}{$\begin{array}{l}\text { Urban } \\
\text { Mines }\end{array}$} & \multirow{3}{*}{$40 / 40$} & \multirow{3}{*}{$\begin{array}{l}\text { Trees: } 54 \\
\text { Building: } 28\end{array}$} & \multirow{3}{*}{25} & $\mathrm{R}$ & $354 \pm 25$ \\
\hline & & & & $\mathrm{S}$ & $455 \pm 60$ \\
\hline & & & & $\mathrm{S}+\mathrm{F}$ & $415 \pm 44$ \\
\hline
\end{tabular}

The synthetic scenario called Field is made by 5 targets scattered over the area, with about 10 targets per group. There are no obstacles. Fig. 4a represents the initial configuration: 80 total drones arranged into 4 swarms are represented by triangular forms, and are placed at the antipodes of the area, whereas the targets are represented by cluster of dots. Fig. $4 \mathrm{~b}$ shows the spatial arrangement of swarms with different form

\footnotetext{
${ }^{1}$ https://ccl.northwestern.edu/netlogo/
} 
and size. Finally Fig. 4c represents three stigmergic formations on three different targets. On the bottom right it can be observed that a stigmergic formation attracted a swarm of drones. Results in Table II confirm that the combination of stigmergy and flocking behaviors speed up the target search process in the Field scenario.
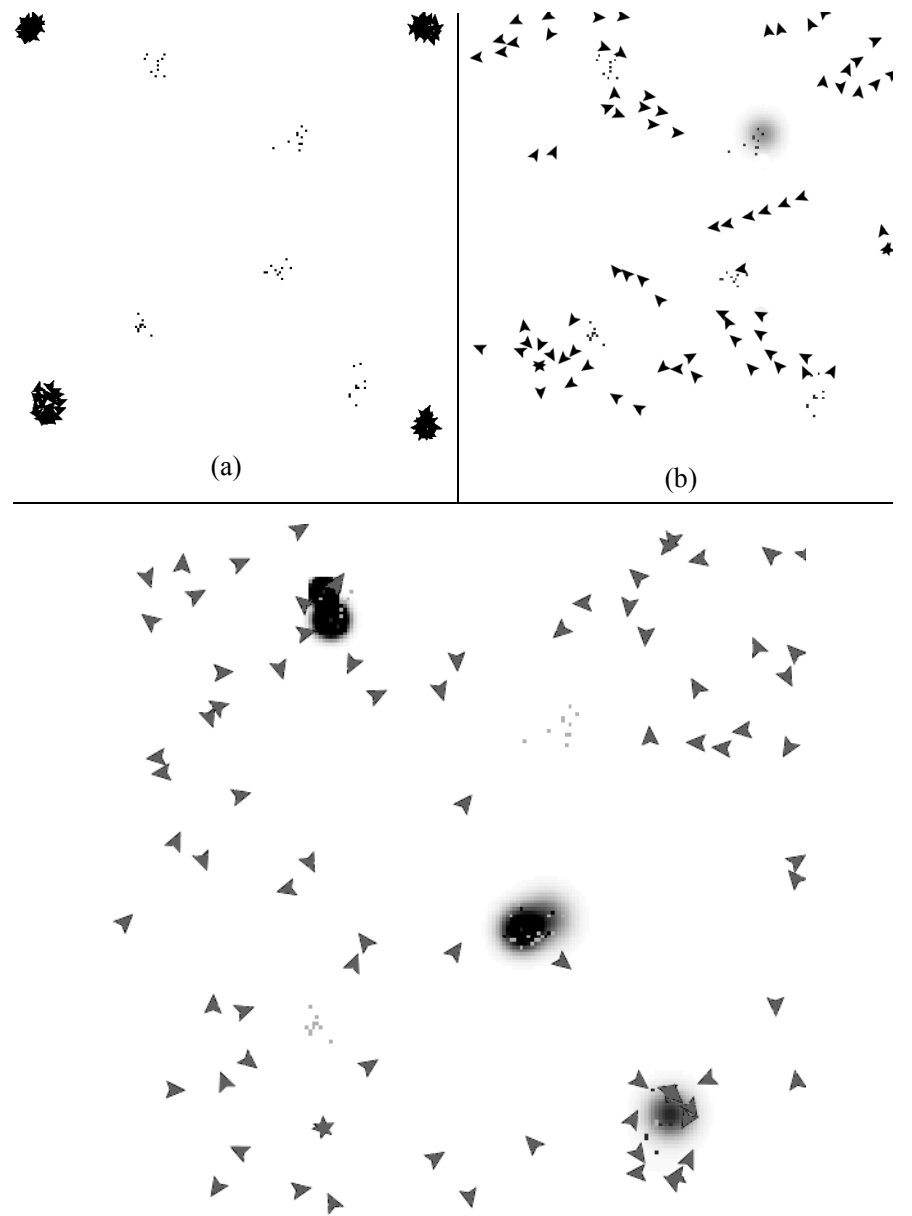

(c)

Fig. 4. Some snapshot of the scenario Field: (a) initial state; (b) flocks; (c) three stigmergic formations.

The Forest scenario (Fig. 5a) represents a synthetic reconstruction of spread targets in a stand of timber. Here, 20 targets and 400 trees are represented by black and gray dots, respectively. 80 total drones, arranged into 4 swarms, have been initially placed at the antipodes of area. Again, the combination of stigmergy and flocking sensibly improves both the mean and the confidence interval.

The Urban scenario (Fig. 5b) is characterized by two cluster of 110 total targets placed on two sides of 7 total buildings. 40 drones, arranged into 4 swarms, are placed at the antipodes of the area, with no trees at all. Also for this scenario, the performance of Table II confirms the positive trend of the approach combining stigmergy and flocking.

The Rural Mines (Fig. 5c-d) and Urban Mines (Fig. 5e-f) scenarios are derived from real-world examples of areas near Sarajevo, in Bosnia-Herzegovina, with landmine objects, selected from publicly available maps ${ }^{2}$. Recently, some authors actually proposed the use of small drones for detecting landmines [12]. On both cases, drones have been initially placed on the boundaries of the area. With respect to the map of the first three scenarios, whose area is 200 square meters, in the last two scenarios the area is 400 square meters. In the Rural Mines scenario, the positive effect of Stigmergy and Flocking is confirmed. Thus, it can be observed in Table II that to adopt Stigmergy, and then Stigmergy with Flock, improves the performance in all scenarios, except for the Urban Mines scenario, whose trend is the opposite. This result could be ascribed to the fact that the Urban Mines map has a very complex layout of obstacles, which makes difficult flock navigation and coordination strategy. It is worth noting that all scenarios have been processed by using a general purpose parameterization. Indeed, a parameterization ad initialization adapted to types of scenario might produce different results.

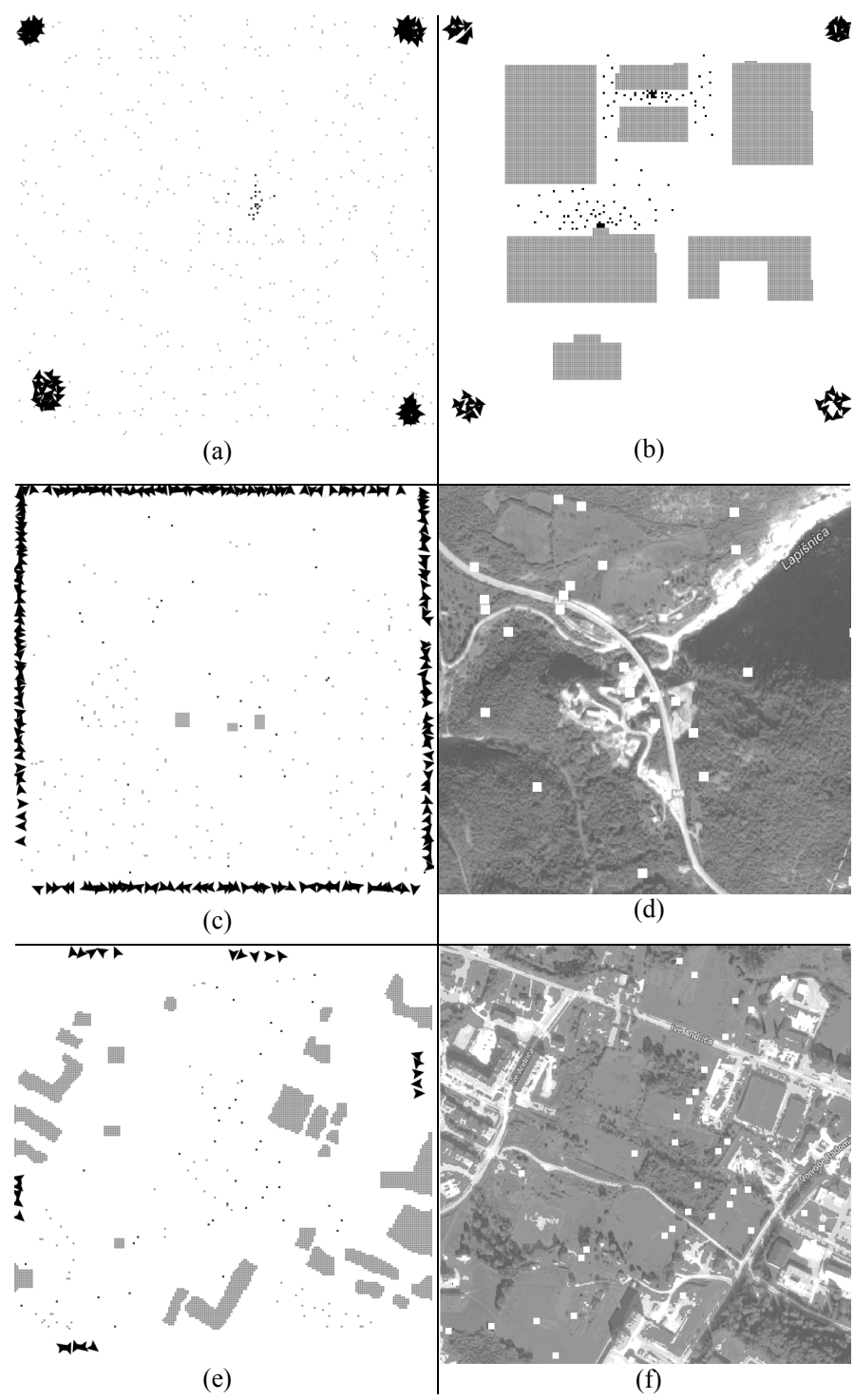

Fig. 5. Models of 2 synthetic and 2 real-world scenarios: (a) Forest; (b) Urban; (c-d) Rural Mines; (e-f) Urban Mines.

${ }^{2}$ http://www.see-demining.org/main.htm 
To better investigate the dynamics of the target search, Fig. 6a-c shows the completion rate against time, for the Urban scenario.

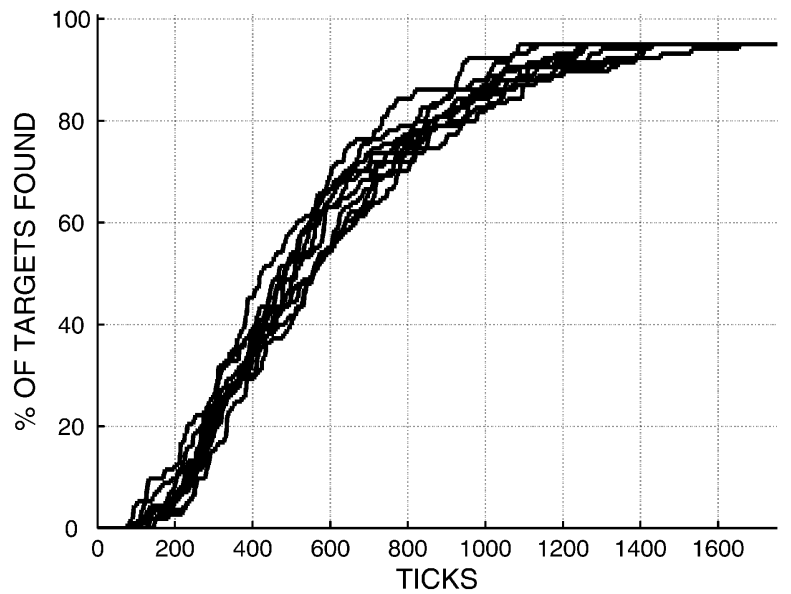

(a)

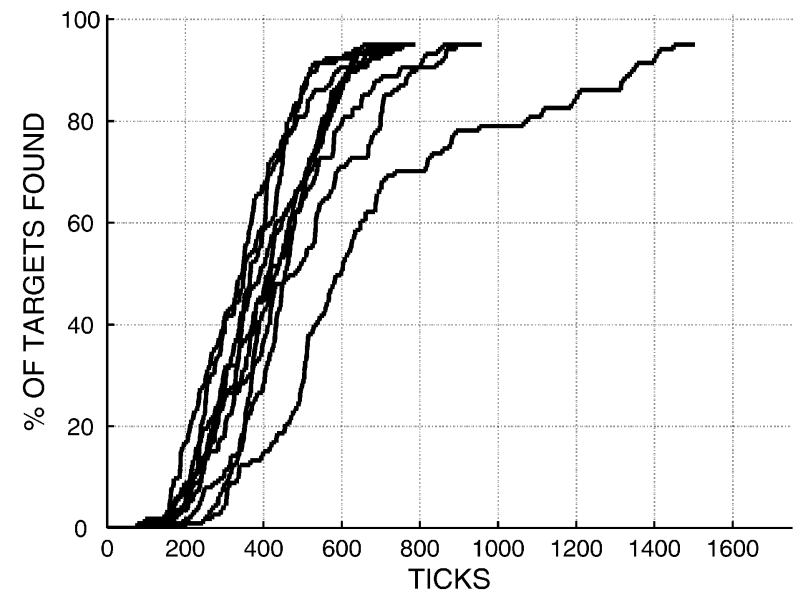

(b)

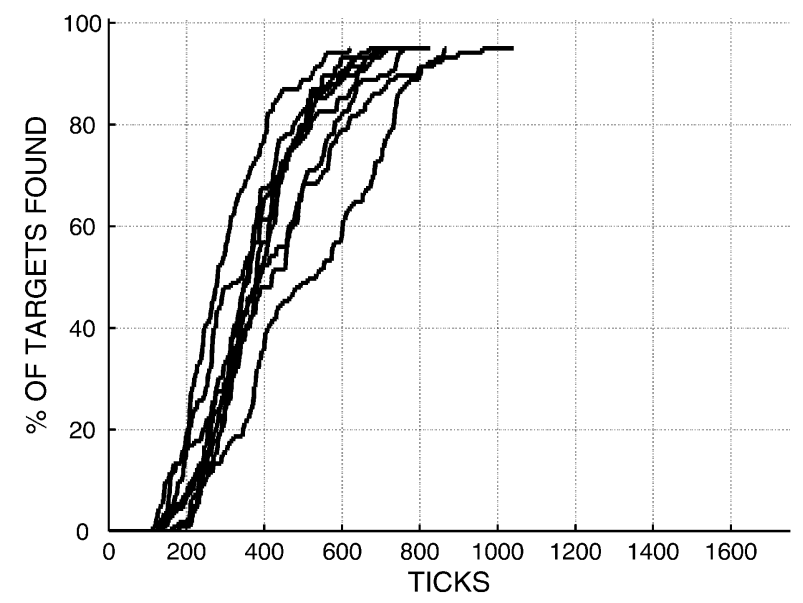

(c)

Fig. 6. Completion rate against time for the Urban scenario: (a) Random Fly; (b) Stigmergy; (c) Stigmergy + Flocking.

Here, the evolution of each trial of an experiment is shown. It is apparent that Stigmergy introduces a significant improvement over Random Fly, starting from about one third of the target coverage, whereas the combination of Stigmergy and Flocking overtakes Stigmergy especially in terms of variability between trials.

\section{CONCLUSIONS AND FUTURE WORKS}

In this paper, we have presented a novel swarm strategy for coordinating small drones performing target search, based on stigmergy and flocking behaviors. The approach uses stigmergy to attract drones in areas with potential targets and employs flocking to organize drones into swarms. Simulated results on synthetic and real-world scenarios proved the benefits of stigmergy and flocking. The overall mechanism can be better enabled if structural parameters are correctly tuned for the given scenario. Determining such correct parameters is not a simple task since different areas have different features. Thus, an appropriate tuning to adapt parameters to the specific search area is desirable to make the search more effective. For this purpose, to use a parameter optimization strategy is considered a key investigation activity for future works.

\section{REFERENCES}

[1] K. Whitehead, C.H. Hugenholtz, S. Myshak, O. Brown, A. LeClair, A Tamminga, T.E. Barchyn, B. Moorman, B. Eaton, "Remote sensing of the environment with small unmanned aircraft systems (UASs), part 2: scientific and commercial applications", Journal of Unmanned Vehicle Systems, vol. 02(03), pp. 86-102, 2014.

[2] R. McCune, R. Purta, M. Dobski, A. Jaworski, G. Madey, Y. Wei, A. Madey \& M.B. Blake, "Investigations of DDDAS for command and control of uav swarms with agent-based modeling", Proceedings of the 2013 Winter Simulation Conference: Simulation: Making Decisions in a Complex World, IEEE Press, pp. 1467-1478, 2013.

[3] M. Brambilla, E. Ferrante, M. Birattari e M. Dorigo, "Swarm robotics: a review from the swarm engineering perspective," Swarm Intelligence, vol. 7, pp. 1-41, 2013.

[4] J. A. Sauter, R. Matthews, H. Van Dyke Parunak \& S. A. Brueckner, "Performance of digital pheromones for swarming vehicle control," in Proceedings of the fourth international joint conference on Autonomous agents and multiagent systems, ACM, pp. 903-910, 2005.

[5] H. Van Dyke Parunak, M. Purcell \& R. O'Connell, «Digital pheromones for autonomous coordination of swarming UAV's," in AIAA's 1st Technical Conference and Workshop on Unmanned Aerospace Vehicles, Ann Arbor, 1001, pp. 48105-1579, 2002.

[6] G. Ermacora, A. Toma, B. Bona, M. Chiaberge, M. Silvagni, "A cloud robotics architecture for an emergency management and monitoring service in a smart city environment," in: 2013 IEEE/RSJ International Conference of Intelligent Robots and Systems, Tokyo (Japan), November 3-8, 2013.

[7] C. W. Reynolds, "Flocks, herds and schools: A distributed behavioral model," in ACM Siggraph Computer Graphics, 1987.

[8] C. Bernon, M.P. Gleizes, \& G. Picard, "Enhancing self-organising emergent systems design with simulation," Engineering Societies in the Agents World VII. Springer Berlin Heidelberg, pp. 284-299, 2007.

[9] T. Kuyucu, I. Tanev \& K. Shimohara, "Superadditive effect of multirobot coordination in the exploration of unknown environments via stigmergy," Neurocomputing, vol. 148, pp. 83-90, 2015.

[10] P. Dasgupta, "A multiagent swarming system for distributed automatic target recognition using unmanned aerial vehicles," IEEE Transactions on Systems, Man, and Cybernetics-Part A: Systems and Humans, Vol. 38, no. 3, pp.549-563, 2008.

[11] Y. Tan \& Z. Zhong-Yang, "Research advance in swarm robotics," Defence Technology 9.1, 18-39, 2013.

[12] J. Rodriguez, C. Castiblanco, I. Mondragon e J. Colorado, "Low-cost quadrotor applied for visual detection of landmine-like objects," in ICUAS - International Conference on Unmanned Aircraft Systems, Orlando, FL, USA, 2014. 